\title{
Serum levels of receptor-interacting protein kinase-3 in patients with COVID-19
}

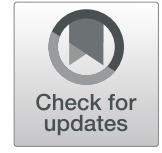

\author{
Hideta Nakamura* ${ }^{*}$, Takeshi Kinjo, Wakako Arakaki, Kazuya Miyagi, Masao Tateyama and Jiro Fujita
}

Keywords: COVID-19, Acute respiratory distress syndrome, Necroptosis, Receptor-interacting kinase 3

\section{Dear Editor:}

Patients with coronavirus disease 2019 (COVID-19) can develop acute respiratory distress syndrome (ARDS), which has been linked to poor prognosis and is a major contributor to patient death [1]. A better understanding of the pathophysiology of COVID-19-related ARDS would benefit early, precise treatment.

Cell death plays a major role in ARDS pathogenesis. While apoptosis in acute lung injury is well studied, newly identified cell death signaling has drawn attention as a potential mediator of ARDS [2]. Necroptosis, a caspaseindependent form of necrosis involving receptorinteracting kinase 3 (RIPK-3), has been implicated in ARDS development with sepsis and trauma [3]. Since this highly regulated cell death signaling leads to rupture of the plasma membrane and release of damage-associated molecular patterns [4], necroptosis may be a therapeutic target for ARDS. However, the relationship between necroptosis and COVID-19-induced ARDS remains unclear.

Here, we describe serum RIPK-3 levels in COVID-19 patients measured on the first day of hospitalization. Patients were recruited from March 1 to May 30, 2020, and diagnosed as "severe" if any of the following conditions were met [5]: (1) respiratory rate $>30$ breaths $/ \mathrm{min}$, (2) saturation of peripheral oxygen $<93 \%$ in ambient air, (3) ratio of arterial partial pressure of oxygen to the fraction of inspired oxygen $<300 \mathrm{mmHg}$, or (4) lung infiltrates $>50 \%$ within 24-48 h. Blood samples were centrifuged within $30 \mathrm{~min}$ and refrigerated at $4{ }^{\circ} \mathrm{C}$, and plasma aliquots were frozen within $12 \mathrm{~h}$. RIPK-3 levels were measured using an enzyme-linked immunosorbent assay (Wuhan Huamei Biotech, Wuhan, China).

This observational study enrolled 16 COVID-19 patients (11 males, 68.8\%) (Table 1). Confirmation of severe acute respiratory syndrome coronavirus 2 (SARS$\mathrm{CoV}-2$ ) infection was by real-time reverse transcription polymerase chain reaction of nasopharyngeal swabs. Patients' median age was 55 years (interquartile range [IQR] 40.5-71.5 years), and the median duration from symptom onset to hospitalization was 7 days (3.259 days). On admission, 14 patients (87.5\%) were confirmed to have COVID-19 pneumonia by chest computed tomography, 10 patients were diagnosed with severe COVID-19 and ARDS, and 6 patients were diagnosed as mild. While hospitalized, the antiviral drug favipiravir was administrated to 11 patients $(68.8 \%)$ in the context of a clinical trial, whereas azithromycin $(n=$ $11,68.8 \%)$, nafamostat $(n=12,75 \%)$, and tocilizumab $(n=7,43.7 \%)$ were commenced as off-label use. The median levels of serum RIPK-3 were significantly higher in severe COVID-19 cases than in mild cases $(483.5 \mathrm{pg} /$ $\mathrm{mL}, \quad$ IQR $\quad 329.6-867.7 \mathrm{pg} / \mathrm{mL}$ vs. $139.9 \mathrm{pg} / \mathrm{mL}, \quad$ IQR 95.37-286.8 pg/mL, $p=0.0075$ ) (Fig. 1). Fifteen patients recovered and were discharged, whereas three patients in the severe group were intubated due to severe acute respiratory failure and one of these patients died.

This is the first study to analyze RIPK-3 in COVID-19 patients. The higher serum RIPK-3 levels in severe patients suggest that RIPK-3-mediated signaling, such as

\footnotetext{
* Correspondence: hnnakamu@med.u-ryukyu.ac.jp

Department of Infectious, Respiratory and Digestive Medicine, Graduate

School of Medicine, University of the Ryukyus, Okinawa, Japan
} 
Table 1 Clinical characteristics of patients $(n=16)$

\begin{tabular}{|c|c|}
\hline Male & $11(68.8 \%)$ \\
\hline Median age, years (IQR) & $55(40.5-71.5)$ \\
\hline Median duration from onset of symptoms to hospitalization, days (IQR) & $7(3.25-9)$ \\
\hline \multicolumn{2}{|l|}{ Underlying disease } \\
\hline Diabetes mellitus & $4(25 \%)$ \\
\hline Hypertension & $2(12.5 \%)$ \\
\hline Heart disease & $2(12.5 \%)$ \\
\hline \multicolumn{2}{|l|}{ Treatment } \\
\hline Azithromycin & $11(68.8 \%)$ \\
\hline Favipiravir & $11(68.8 \%)$ \\
\hline Nafamostat & $12(75 \%)$ \\
\hline Tocilizumab & $7(43.7 \%)$ \\
\hline \multicolumn{2}{|l|}{ Disease severity } \\
\hline Mild & $6(37.5 \%)$ \\
\hline Severe & $10(62.5 \%)$ \\
\hline \multicolumn{2}{|l|}{$\mathrm{PaO}_{2} / \mathrm{FiO}_{2}$ ratio } \\
\hline$>350$ & $6(37.5 \%)$ \\
\hline 200-300 & $5(31.25 \%)$ \\
\hline $150-200$ & $3(18.75 \%)$ \\
\hline$<150$ & $2(12.5 \%)$ \\
\hline
\end{tabular}

IQR interquartile range, $\mathrm{PaO}_{2} / \mathrm{FiO}_{2}$ ratio ratio of arterial partial pressure of oxygen to the fraction of inspired oxygen

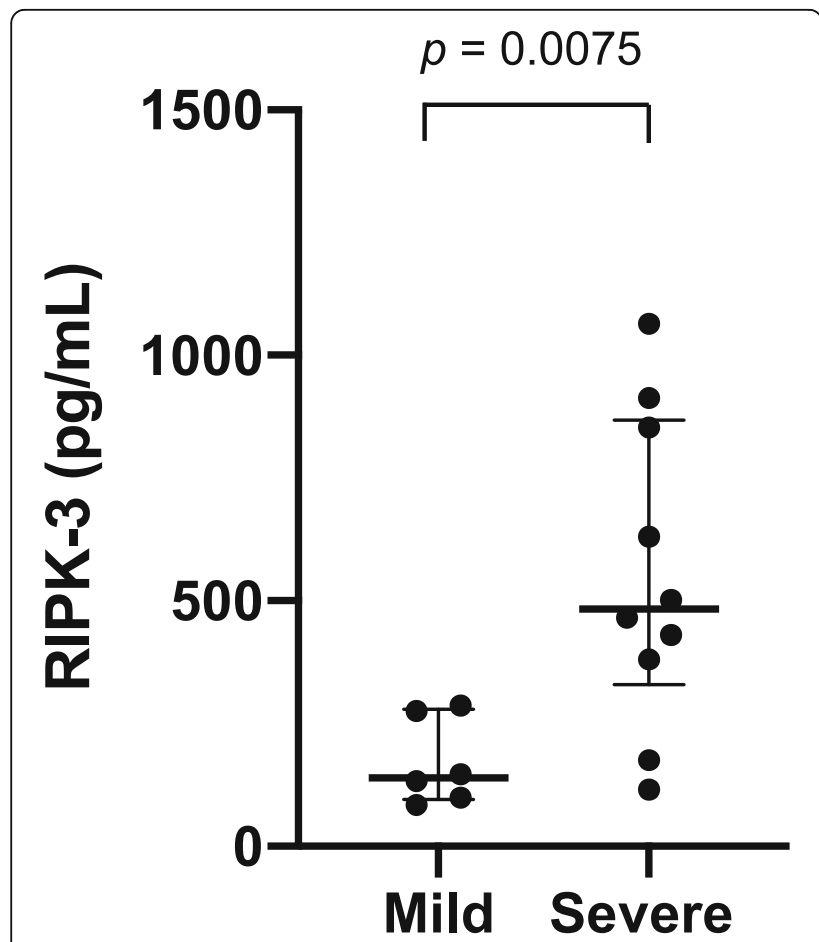

Fig. 1 Serum levels of receptor-interacting kinase 3 (RIPK-3) in 16 patients with COVID-19. Serum RIPK-3 levels were measured by the enzyme-linked immunosorbent assay in patients with mild $(n=6)$ or severe $(n=10)$ COVID-19. For each dataset, the horizontal bars represent the median and interquartile range. Statistics were analyzed using Prism (GraphPad Software, CA, USA); $p<0.05$ was considered significant necroptosis, might be involved in the development of acute lung injury associated with COVID-19 pneumonia. Siempos et al. reported plasma RIPK-3 levels were significantly higher in ARDS patients compared to those of non-ARDS patients [6]. Shashaty et al. demonstrated that among patients with sepsis or trauma, the change in plasma RIPK-3 levels $48 \mathrm{~h}$ after admission was independently associated with ARDS [3]. Because RIPK-3 mediates not only necroptosis but also other inflammatory pathways [2], the elevation of RIPK-3 does not directly indicate the execution of necroptosis. To confirm the role of RIPK-3 in COVID-19-ARDS patients, further studies are needed including a larger number of participants and histological evaluation of lung tissues, especially since RIPK-3-mediated necroptosis could be a potential therapeutic target for COVID19-related ARDS.

\section{Abbreviations}

COVID-19: Coronavirus disease 2019; ARDS: Acute respiratory distress syndrome; RIPK1/3: Receptor-interacting kinase 1 and 3 (RIPK1/3); IQR: Interquartile range; SARS-CoV-2: Severe acute respiratory syndrome coronavirus 2

\section{Acknowledgements}

Collaborating author names: Mariko Otsuki, Yuri Higure, Naoya Nishiyama, Masashi Nakamatsu, and Shusaku Haranaga. Department of Infectious, Respiratory and Digestive Medicine, Graduate School of Medicine, University of the Ryukyus, Okinawa, Japan. 


\section{Authors' contributions}

HN designed the study and had full access to all data in the study. TK and WA contributed to the laboratory work. MT and JF supervised the study and contributed to the writing of the manuscript. All authors contributed to data acquisition, data analysis, or data interpretation and reviewed and approved the final version of the manuscript.

\section{Funding}

Not applicable.

\section{Availability of data and materials}

Full de-identified data of the analyses are available upon request sent to the corresponding author.

\section{Ethics approval and consent to participate}

This study was approved by the ethics committee of the University of the Ryukyus for Medical and Health Research Involving Human Subjects (approval number: 1616)

\section{Consent for publication}

Written informed consent was obtained from all patients.

\section{Competing interests}

Not applicable.

Received: 17 July 2020 Accepted: 28 July 2020

Published online: 04 August 2020

\section{References}

1. Cao Y, Liu X, Xiong L, Xiong L, Cai K. Imaging and clinical features of patients with 2019 novel coronavirus SARS-CoV-2: a systematic review and meta-analysis. J Med Virol. 2020;3:https://doi.org/10.1002/jmv.25822.

2. Faust $\mathrm{H}$, Mangalmurti NS. Collateral damage: necroptosis in the development of lung injury. Am J Physiol Lung Cell Mol Physiol. 2020; 318(2):L215-25.

3. Shashaty MGS, Reilly JP, Faust HE, Forker CM, Glttner CA, Zhang PX, et al. Plasma receptor interacting protein kinase-3 levels are associated with acute respiratory distress syndrome in sepsis and trauma: a cohort study. Crit Care. 2019;23(1):235

4. Linkermann A, Green DR. Necroptosis. N Engl J Med. 2014;30(5):455-65.

5. Wu Z, McGoogan JM. Characteristics of and important lessons from the coronavirus disease 2019 (COVID-19) outbreak in China: summary of a report of 72314 cases from the Chinese Center for Disease Control and Prevention. JAMA. 2020;24. https://doi.org/10.1001/jama.2020.2648.

6. Siempos II, Ma KC, Imamura M, Baron RM, Fredenburgh LE, Huh JW, et al. RIPK3 mediates pathogenesis of experimental ventilator-induced lung injury. JCl Insight. 2018;3(9):e97102.

\section{Publisher's Note}

Springer Nature remains neutral with regard to jurisdictional claims in published maps and institutional affiliations. 